\title{
Implications of Yukawa texture in the charged Higgs boson phenomenology within 2HDM-III
}

\section{A. Cordero-Cid}

Fac. de Cs. de la Electrónica, Benemérita Universidad Autónoma de Puebla, Apdo. Postal 542,

Puebla, Pue. 72570, México.

\section{O. Félix-Beltrán}

Fac. de Cs. de la Electrónica, Benemérita Universidad Autónoma de Puebla, Apdo. Postal 542,

Puebla, Pue. 72570, México.

\section{Jaime Hernández-Sánchez ${ }^{* \dagger}$}

Fac. de Cs. de la Electrónica, Benemérita Universidad Autónoma de Puebla, Apdo. Postal 542,

Puebla, Pue. 72570, México.

Dual C-P Institute of High Energy Physics, Puebla, Pue., México.

E-mail: jaimeh@ece.buap.mx

\section{R. Noriega-Papaqui}

Dual C-P Institute of High Energy Physics, Puebla, Pue., México.

Área Académica de Matemáticas y Física, Universidad Autónoma del Estado de Hidalgo, Carr.

Pachuca-Tulancingo Km. 4.5, Pachuca, Hgo. 42184, México.

We discuss the implications of assuming a four-zero Yukawa texture for the properties of the charged Higgs boson within the context of the general 2-Higgs Doublet Model of Type III. We present the charged Higgs boson couplings with heavy quarks and the resulting pattern for its decays, including the decay $H^{+} \rightarrow W^{+} \gamma$ at 1-loop level. The parameters chosen can still avoid the $B \rightarrow X_{s} \gamma$ constraint, the perturbativity and $\rho_{0}$ bound. Also, we present the constraints of $B 0-\bar{B} 0$ mixing and of the radiative corrections to the $Z b \bar{b}$ vertex in the regime small $\tan \beta$. The production of charged Higgs bosons is also sensitive to the modifications of its couplings, so that we also evaluate the resulting effects on 'direct' $c \bar{b} \rightarrow H^{+}+$c.c. and 'indirect' $q \bar{q}, g g \rightarrow \bar{t} b H^{+}+$c.c. production. Significant scope exists at the Large Hadron Collider for several $H^{ \pm}$production and decay channels combined to enable one to distinguish between such a model and alternative 2Higgs doublet scenarios.

Third International Workshop on Prospects for Charged Higgs Discovery at Colliders - CHARGED2010, September 27-30, 2010

Uppsala Sweden

\footnotetext{
${ }^{*}$ Speaker.

${ }^{\dagger}$ SNI-CONACYT and PROMEP-SEP grants
} 


\section{Introduction}

The 2HDM-II has been quite attractive to date, in part because it coincides with the Higgs sector of the MSSM, wherein each Higgs doublet couples to the $u$ - or $d$-type fermions separately ${ }^{1}$. However, this is only valid at tree-level [2]. Thus, we can consider the 2HDM-III as a generic description of physics at a higher scale (of order $\mathrm{TeV}$ or maybe even higher), whose low energy imprints are reflected in the Yukawa coupling structure. With this idea in mind, a detailed study of the 2HDM-III Yukawa Lagrangian was presented in Refs.[3, 4], under the assumption of a specific texture pattern [5], which generalizes the original model of Ref. [6]. The extension of such an approach to investigate charged Higgs boson phenomenology was conducted in Ref. [4, 7], which discussed the implications of this Yukawa texture for the charged Higgs boson properties (masses and couplings) and the resulting pattern of charged Higgs boson decays and main production reactions at the LHC.

This paper is organized as follows. In section 2, we discuss the Higgs-Yukawa sector of the 2HDM-III. Then, in section 3, we shows the BR of the decays of the charged Higgs boson, including $H^{+} \rightarrow W^{+} \gamma$ at one-loop level. Actual LHC event rates for the main production mechanisms at the LHC are given in section 4. These include the $s$-channel production of charged Higgs bosons through $c \bar{b}(\bar{c} b)$-fusion and the multi-body more $q \bar{q}, g g \rightarrow t \bar{b} H^{-}+$c.c. (charge conjugated). Finally, we summarize our results and present the conclusions in section 5 .

\section{The charged Higgs boson Lagrangian and the fermionic couplings}

In order to derive the interactions of the charged Higgs boson, the Yukawa Lagrangian is given by:

$$
\mathscr{L}_{Y}=Y_{1}^{u} \bar{Q}_{L} \tilde{\Phi}_{1} u_{R}+Y_{2}^{u} \bar{Q}_{L} \tilde{\Phi}_{2} u_{R}+Y_{1}^{d} \bar{Q}_{L} \Phi_{1} d_{R}+Y_{2}^{d} \bar{Q}_{L} \Phi_{2} d_{R}
$$

where $\Phi_{1,2}=\left(\phi_{1,2}^{+}, \phi_{1,2}^{0}\right)^{T}$ refer to the two Higgs doublets, $\tilde{\Phi}_{1,2}=i \sigma_{2} \Phi_{1,2}^{*}, Q_{L}$ denotes the lefthanded fermion doublet, $u_{R}$ and $d_{R}$ are the right-handed fermions singlets and, finally, $Y_{1,2}^{u, d}$ denote the $(3 \times 3)$ Yukawa matrices. Similarly, one can write the corresponding Lagrangian for leptons.

After spontaneous EWSB and including the diagonalizing matrices for quarks and Higgs bosons ${ }^{2}$, through rotated matrices $\tilde{Y}_{n}^{q}=V_{q} Y_{n}^{q} V_{q}^{\dagger}$ ( $n=1$ when $q=u$, and $n=2$ when $q=d$ ) where $V_{q}$ is the diagonalizing mass matrix. One can derive a better approximation for the product $V_{q} Y_{n}^{q} V_{q}^{\dagger}$, expressing the rotated matrix $\tilde{Y}_{n}^{q}$, in the form

$$
\left[\tilde{Y}_{n}^{q}\right]_{i j}=\frac{\sqrt{m_{i}^{q} m_{j}^{q}}}{v}\left[\tilde{\chi}_{n}^{q}\right]_{i j}=\frac{\sqrt{m_{i}^{q} m_{j}^{q}}}{v}\left[\chi_{n}^{q}\right]_{i j} e^{i \vartheta_{i j}^{q}},
$$

where $\chi$ 's are unknown dimensionless parameters of the model, they come from the election of a specific texture of the Yukawa matrices. We find the Lagrangian of the interactions of the charged

\footnotetext{
${ }^{1}$ Notice that there exist significant differences between the 2HDM-II and MSSM though, when it comes to their mass/coupling configurations and possible Higgs signals [1].

${ }^{2}$ The details of both diagonalizations are presented in Ref. [3].
} 
Higss boson with quark pairs as follows:

$$
\begin{aligned}
\mathscr{L}^{q}= & \frac{g}{2 \sqrt{2} M_{W}} \sum_{l=1}^{3} \bar{u}_{i}\left\{\left(V_{\mathrm{CKM}}\right)_{i l}\left[\tan \beta m_{d_{l}} \delta_{l j}-\frac{\sec \beta}{\sqrt{2}} \sqrt{m_{d_{l}} m_{d_{j}}} \tilde{\chi}_{l j}^{d}\right]\right. \\
& +\left[\cot \beta m_{u_{i}} \delta_{i l}-\frac{\csc \beta}{\sqrt{2}} \sqrt{m_{u_{i}} m_{u_{l}}} \tilde{\chi}_{i l}^{u}\right]\left(V_{\mathrm{CKM}}\right)_{l j} \\
& +\left(V_{\mathrm{CKM}}\right)_{i l}\left[\tan \beta m_{d_{l}} \delta_{l j}-\frac{\sec \beta}{\sqrt{2}} \sqrt{m_{d_{l}} m_{d_{j}}} \tilde{\chi}_{l j}^{d}\right] \gamma^{5} \\
& \left.-\left[\cot \beta m_{u_{i}} \delta_{i l}-\frac{\csc \beta}{\sqrt{2}} \sqrt{m_{u_{i}} m_{u_{l}}} \tilde{\chi}_{i l}^{u}\right]\left(V_{\mathrm{CKM}}\right)_{l j} \gamma^{5}\right\} d_{j} H^{+},
\end{aligned}
$$

where we have redefined $\left[\tilde{\chi}_{1}^{u}\right]_{i j}=\tilde{\chi}_{i j}^{u}$ and $\left[\tilde{\chi}_{2}^{d}\right]_{i j}=\tilde{\chi}_{i j}^{d}$. Then, from Eq. (2.3), the couplings $\bar{u}_{i} d_{j} H^{+}$ and $u_{i} \bar{d}_{j} H^{-}$are given by: $g_{H^{+} \bar{u}_{i} d_{j}}=-\frac{i g}{2 \sqrt{2} M_{W}}\left(S_{i j}+P_{i j} \gamma_{5}\right), g_{H^{-} u_{i} \bar{d}_{j}}=-\frac{i g}{2 \sqrt{2} M_{W}}\left(S_{i j}-P_{i j} \gamma_{5}\right)$, where $S_{i j}$ and $P_{i j}$ are defined as: ${ }_{P_{i j}}^{S_{i j}}=\sum_{l=1}^{3}\left(V_{\mathrm{CKM}}\right)_{i l} m_{d_{l}} X_{l j} \pm m_{u_{i}} Y_{i l}\left(V_{\mathrm{CKM}}\right)_{l j}$. with $X_{l j}=\left[\tan \beta \delta_{l j}-\right.$ $\left.\frac{\sec \beta}{\sqrt{2}} \sqrt{\frac{m_{d_{j}}}{m_{d_{l}}}} \tilde{\chi}_{l j}^{d}\right], Y_{i l}=\left[\cot \beta \delta_{i l}-\frac{\csc \beta}{\sqrt{2}} \sqrt{\frac{m_{u_{l}}}{m_{u_{i}}}} \tilde{\chi}_{i l}^{u}\right]$. Based on the analysis of $B \rightarrow X_{s} \gamma[8,9]$, it is claimed that $X \leq 20$ and $Y \leq 1.7$ for $m_{H^{+}}>250 \mathrm{GeV}$, while for a lighter charged Higgs boson mass, $m_{H^{+}} \sim 180 \mathrm{GeV}$, one gets $(X, Y) \leq(18,0.5)$. Thus, we find the bounds: $\left|\chi_{33}^{u, d}\right| \lesssim 1$ for $0.1<$ $\tan \beta \leq 70$ [4]. On the other hand, the condition $\frac{\Gamma_{H^{+}}}{m_{H^{+}}}<\frac{1}{2}$ in the frame of the 2HDM-III implies $\frac{\Gamma_{H^{+}}}{m_{H^{+}}} \approx \frac{3 G_{F} m_{t}^{2}}{4 \sqrt{2} \pi \tan \beta^{2}}\left(\frac{1}{1-\frac{\tilde{\chi}_{33}^{u}}{\sqrt{2} \cos \beta}}\right)^{2}$, we have checked numerically that this leads to $0.08<\tan \beta<200$ when $\left|\tilde{\chi}_{33}^{u}\right| \approx 1$ and $0.3<\tan \beta<130$ as long as $\left|\tilde{\chi}_{33}^{u}\right| \rightarrow 0$ recovering the result for the case of the 2HDM-II [10].

Another important bounds on $\left|\tilde{\chi}_{33}\right|$ and $\tan \beta$ comes from radiative corrections to the process $\Gamma(Z \rightarrow b \bar{b})$, specially the hadronic branching fraction of $Z$ bosons to $b \bar{b}\left(R_{b}\right)$ and the $b$ quark asymmetry $\left(A_{b}\right)$ impossed a high restriction. Then, following the calculation of the Ref. [7], in the regime of $\operatorname{small} \tan \beta$, we can find bounds for $\tan \beta$ : in the case $\chi_{33}^{u, d}=1$ and $m_{H^{+}} \sim 200(300) \mathrm{GeV}$, the range $\tan \beta>0.3(0.2)$ is allowed, while in the scenario $\chi_{33}^{u, d}=-1$ and $m_{H^{+}} \sim 200(300) \mathrm{GeV}$, $\tan \beta>5(3)$ is permitted.

In the Ref. [7] is presented the analysis of the quantity that parameterizes the $B_{0}-\overline{B_{0}}$ mixing: $x_{d} \equiv \frac{\Delta m_{B}}{\Gamma_{B}}$, where we obtain bounds for $\tan \beta$ and mass of the charged Higgs boson. Combining the criteria of the analysis radiative corrections of $Z b \bar{b}$ vertex and $B_{0}-\bar{B}_{0}$ mixing, $\tan \beta>0.3$ is allowed for $m_{H^{+}}>170 \mathrm{GeV}$ and $\chi_{33}^{u, d}=1$. However, when $\chi_{33}^{u, d}=-1$ and $m_{H^{+}}<600 \mathrm{GeV}$, $\tan \beta<2$ is disfavored.

Besides, following the analysis of the Ref. [7], one can get the deviation $\Delta \rho_{0}$ of the parameter $\rho_{0}=M_{W}^{2} / \rho M_{Z}^{2} C_{W}^{2}$ of our version 2HDM-III, where the $\rho$ in the denominator absorbs all the SM corrections, among which the most important SM correction at 1-loop level comes from the heavy top-quark. We can get that for the case $\alpha=0, \pi / 2$, the parameter space of the scalar sector is strongly reduced when decoupling between Higgs bosons, i.e. $\Delta m_{i j}=m_{i}-m_{j}>100 \mathrm{GeV}\left(m_{i}=\right.$ $\left.m_{h^{0}}, m_{H^{0}}, m_{A^{0}}, m_{H^{ \pm}}\right)$. However, is possible to avoid the constraint for $\Delta \rho_{2 \mathrm{HDM}-\mathrm{III}}$ if the decoupling source $\Delta m_{i j} \sim 20 \mathrm{GeV}$ o $\Delta m_{i j} \sim 100 \mathrm{GeV}$ but one Higgs very heavy (e.g. $m_{H^{0}}>1 \mathrm{TeV}$ ). When $\alpha=\beta \pm \pi / 2$ the allowed parameter region is larger and one can avoid the constraints of the $\rho$ parameter with or without decoupling. 

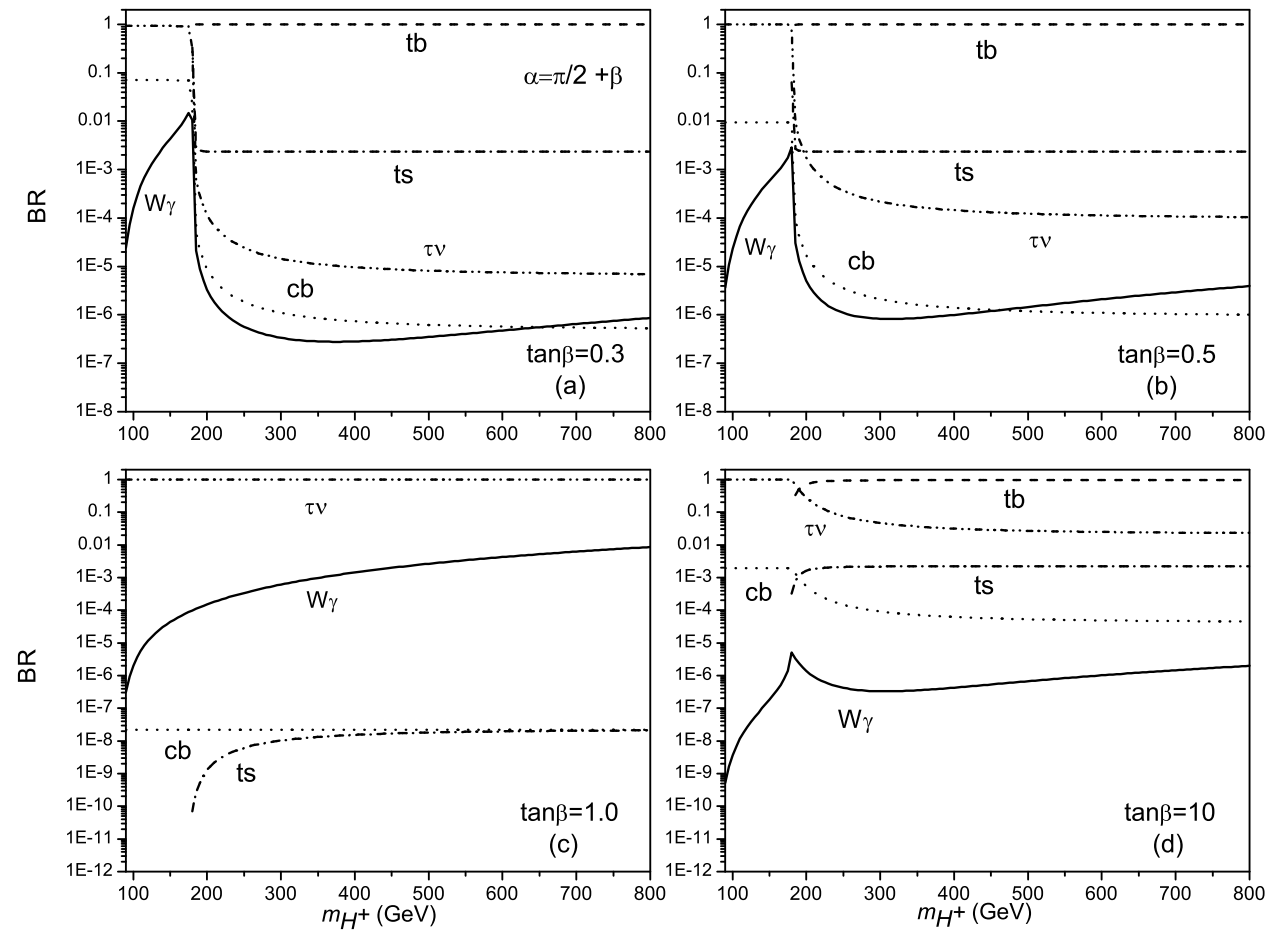

Figure 1: The figure shows the BRs of the $H^{+}$decaying into the principal modes, taking $\tilde{\chi}_{i j}^{u}=1, \tilde{\chi}_{i j}^{d}=1$, $m_{h^{0}}=120 \mathrm{GeV}, m_{A^{0}}=m_{H^{+}}$and $\alpha=\pi / 2+\beta$ for: (a) $\tan \beta=0.3$, (b) $\tan \beta=0.5$, (c) $\tan \beta=1$, (d) $\tan \beta=10$. The lines in each graph correspond to: $\left(W \gamma\right.$ line) $\mathrm{BR}\left(H^{+} \rightarrow W^{+} \gamma\right),(t b$ line $) \mathrm{BR}\left(H^{+} \rightarrow t \bar{b}\right),(c b$ line) $\mathrm{BR}\left(H^{+} \rightarrow c \bar{b}\right),\left(t s\right.$ line) $\mathrm{BR}\left(H^{+} \rightarrow t \bar{s}\right),\left(\tau v\right.$ line) $\mathrm{BR}\left(H^{+} \rightarrow \tau^{+} v_{\tau}\right)$, (Wh line) $\mathrm{BR}\left(H^{+} \rightarrow W^{+} h^{0}\right)$.

\section{Decays of the charged Higgs boson at tree level}

Let us now discuss the decay modes of the charged Higgs boson within our model. Hereafter, we shall refer to scenario with $\tilde{\chi}_{i j}^{u}=1, \tilde{\chi}_{i j}^{d}=1$ and $\tan \beta=0.3,0.5,1,10$. We have performed the numerical analysis of charged Higgs boson decays, taking the mixing angle $\alpha=\pi / 2+\beta$ and varying the charged Higgs boson mass within the interval $100 \mathrm{GeV} \leq m_{H^{+}} \leq 800 \mathrm{GeV}$, further fixing $m_{h^{0}}=120 \mathrm{GeV}, m_{A^{0}} \sim m_{H^{+}}$[7].

We present this special and interesting case in the Fig. 1. For $\tan \beta=0.3$, we show in plot Fig. 1(a) that the relevant channel decay is the $\tau^{+} v_{\tau}$ when $m_{H^{+}}<180 \mathrm{GeV}$, and for the range $m_{H^{+}}>$ $180 \mathrm{GeV}$ the mode $t \bar{b}$ becomes dominant. Here, the BR of the decay $H^{+} \rightarrow W^{+} \gamma$ is relatively large of order $10^{-2}$. From Fig. 1 (b) we can observe that the dominant decay mode is into $\tau^{+} v_{\tau}$ for the range $m_{H^{+}}<175 \mathrm{GeV}$, again for $175 \mathrm{GeV}<m_{H^{+}}<180 \mathrm{GeV}$ the mode $t \bar{s}$ is the leading one, while the mode $W^{+} \gamma$ induced at one-loop level has a BR of order $10^{-3}$. When $m_{H^{+}}>180 \mathrm{GeV}$ the mode $t \bar{b}$ is the leading one. The most interesting case is when $\tan \beta=1$ where the width decay of the mode $t \bar{b}$ is zero. We present this case in the Fig. 1 (c), where one can see that the dominant 
decay is the mode $\tau^{+} v_{\tau}$ for all $m_{H^{+}}$. Besides, the BR $\left(H^{+} \rightarrow W^{+} \gamma\right)$ is of order $10^{-2}$ to $10^{-4}$ for $m_{H^{+}}>180 \mathrm{GeV}$. Now, from Fig. 1(c), where $\tan \beta=10$, we find that the dominant decay mode is into $\tau^{+} v_{\tau}$ for the range $m_{H^{+}}<180 \mathrm{GeV}$. For $180 \mathrm{GeV}<m_{H^{+}}$, the dominant decay of the charged Higgs boson is the mode $t \bar{b}$. We observe that the mode $W^{+} \gamma$ is important when $170 \mathrm{GeV}<m_{H^{+}}<$ $180 \mathrm{GeV}$ and for $0.1 \leq \tan \beta \leq 1$, taking $\tilde{\chi}_{i j}^{u, d}=1$.

\section{Event rates of charged Higgs bosons at the LHC}

To illustrate the type of charged Higgs signatures that have the potential to be detectable at the LHC in the 2HDM-III, we show in Tabs. 1 and 2 the event rates of charged Higgs boson through the channels $q \bar{q}, g g \rightarrow t \bar{b} H_{i}^{-}+$c.c. and $c \bar{b} \rightarrow H^{+}+$c.c., alongside the corresponding production cross sections $(\sigma$ 's) and relevant BRs, for a combination of masses, $\tan \beta$ and specific 2 HDM-III parameters amongst those used in the previous works [4, 7] (assuming $m_{h^{0}}=120 \mathrm{GeV}, m_{A^{0}}=300$ $\mathrm{GeV}$ and the mixing angle at $\alpha=\pi / 2$ throughout). In particular, we focus on those cases where the charged Higgs boson mass is above the threshold for $t \rightarrow b H^{+}$. (As default, we also assume an integrated luminosity of $10^{5} \mathrm{pb}^{-1}$.)

To illustrate these results, let us comment on one case within each scenario. From Table 1, we can see that for Scenario with $\left(\tilde{\chi}_{i j}^{u}=1, \tilde{\chi}_{i j}^{d}=1\right)$ and $\tan \beta=15$, we have that the $H^{ \pm}$is heavier than $m_{t}-m_{b}$, as we take a mass $m_{H^{+}}=400$, thus precluding top decay contributions, so that in this case $\sigma\left(p p \rightarrow t \bar{b} H^{+}\right) \approx 2.2 \times 10^{-1} \mathrm{pb}$, while the dominant decays are $H^{+} \rightarrow t \bar{b}, \tau^{+} v_{\tau} W^{+} h^{0}, W^{+} A^{0}$ which give a number of events of 7040, 46, 13860, 374, respectively. In this case the most promising signal is $H^{+} \rightarrow W^{+} h^{0}$. However, when $\tan \beta=70$ we have that all event rates increase substantially. Here, the signal $H^{+} \rightarrow W^{+} h^{0}$ is still the most important with an event rate of 15480 .

All these rates correspond to the case of indirect production. The contribution due to direct production is in fact subleading, especially at large $m_{H^{ \pm}}$values. Nonetheless, in some benchmark cases, they could represent a sizable addition to the signal event rates. This is especially the case for $\tan \beta=15$ or 70 . In general though, also considering the absence of an accompanying trigger alongside the $H^{ \pm}$,i.e. for instance a top quark produced in $g b \rightarrow H^{-} t$ could help to identify the signal. Thus, we expect that the impact of $c \bar{b}$-fusion at the LHC will be more marginal that of $g g$-fusion for large Higgs masses, in fact, at times even smaller that the contribution from $q \bar{q}$ annihilation [4].

Table 1: Summary of LHC event rates for some parameter combinations with $\left(\tilde{\chi}_{i j}^{u}=1, \tilde{\chi}_{i j}^{d}=1\right)$ and integrated luminosity of $10^{5} \mathrm{pb}^{-1}$, for several different signatures, through the channel $q \bar{q}, g g \rightarrow \bar{t} b H^{+}+$c.c.

\begin{tabular}{|c|c|c|c|c|c|}
\hline$\left(\tilde{\chi}_{i j}^{u}, \tilde{\chi}_{i j}^{d}\right)$ & $\tan \beta$ & $m_{H^{+}}(\mathrm{GeV})$ & $\sigma\left(p p \rightarrow H^{+} \bar{t} b\right)(\mathrm{pb})$ & Relevant BRs & Nr. Events \\
\hline$(1,1)$ & 15 & 400 & $2.23 \times 10^{-1}$ & $\begin{array}{l}\operatorname{BR}\left(H^{+} \rightarrow t \bar{b}\right) \approx 3.2 \times 10^{-1} \\
\operatorname{BR}\left(H^{+} \rightarrow \tau^{+} v_{\tau}^{0}\right) \approx 2.1 \times 10^{-3} \\
\operatorname{BR}\left(H^{+} \rightarrow W^{+} h^{0}\right) \approx 6.3 \times 10^{-1} \\
\operatorname{BR}\left(H_{2}^{+} \rightarrow W^{+} A^{0}\right) \approx 1.7 \times 10^{-2}\end{array}$ & $\begin{array}{r}7040 \\
46 \\
13860 \\
374\end{array}$ \\
\hline$(1,1)$ & 70 & 400 & $4.3 \times 10^{-1}$ & $\begin{array}{l}\operatorname{BR}\left(H^{+} \rightarrow t \bar{b}\right) \approx 3.5 \times 10^{-1} \\
\operatorname{BR}\left(H^{+} \rightarrow c \bar{b}\right) \approx 1.4 \times 10^{-2} \\
\operatorname{BR}\left(H^{+} \rightarrow \tau^{+} v_{\tau}\right) \approx 2.5 \times 10^{-1} \\
\operatorname{BR}\left(H^{+} \rightarrow W^{+} h^{0}\right) \approx 3.6 \times 10^{-1}\end{array}$ & $\begin{array}{r}15050 \\
602 \\
10750 \\
15480\end{array}$ \\
\hline
\end{tabular}


Table 2: Summary of LHC event rates for some parameter combinations with $\left(\tilde{\chi}_{i j}^{u}=1, \tilde{\chi}_{i j}^{d}=1\right)$ and integrated luminosity of $10^{5} \mathrm{pb}^{-1}$, for several different signatures, through the channel $c \bar{b} \rightarrow H^{+}+$c.c.

\begin{tabular}{|c|c|c|c|c|c|}
\hline$\left(\tilde{\chi}_{i j}^{u}, \tilde{\chi}_{i j}^{d}\right)$ & $\tan \beta$ & $m_{H^{+}}(\mathrm{GeV})$ & $\sigma\left(p p \rightarrow H^{+}+X\right)(\mathrm{pb})$ & Relevant BRs & Nr. Events \\
\hline \multirow{4}{*}{$(1,1)$} & \multirow{4}{*}{15} & \multirow{4}{*}{400} & \multirow{4}{*}{$1.14 \times 10^{-1}$} & $\overline{\mathrm{BR}}\left(H^{+} \rightarrow t \bar{b}\right) \approx 3.2 \times 10^{-1}$ & 3648 \\
\hline & & & & $\mathrm{BR}\left(H^{+} \rightarrow \tau^{+} v_{\tau}^{0}\right) \approx 2.1 \times 10^{-3}$ & 24 \\
\hline & & & & $\operatorname{BR}\left(H^{+} \rightarrow W^{+} h^{0}\right) \approx 6.3 \times 10^{-1}$ & 7182 \\
\hline & & & & $\operatorname{BR}\left(H_{2}^{+} \rightarrow W^{+} A^{0}\right) \approx 1.7 \times 10^{-2}$ & 194 \\
\hline \multirow{4}{*}{$(1,1)$} & \multirow{4}{*}{70} & \multirow{4}{*}{400} & \multirow{4}{*}{$1.25 \times 10^{-1}$} & $\mathrm{BR}\left(H^{+} \rightarrow t \bar{b}\right) \approx 3.5 \times 10^{-1}$ & 4375 \\
\hline & & & & $\mathrm{BR}\left(H^{+} \rightarrow c \bar{b}\right) \approx 1.4 \times 10^{-2}$ & 175 \\
\hline & & & & $\operatorname{BR}\left(H^{+} \rightarrow \tau^{+} v_{\tau}\right) \approx 2.5 \times 10^{-1}$ & 3125 \\
\hline & & & & $\operatorname{BR}\left(H^{+} \rightarrow W^{+} h^{0}\right) \approx 3.6 \times 10^{-1}$ & 4500 \\
\hline
\end{tabular}

\section{Conclusions}

We have discussed the implications of assuming a four-zero Yukawa texture for the properties of the charged Higgs boson, within the context of a 2HDM-III. The latter clearly reflect the different coupling structure of the 2HDM-III, e.g., with respect to the 2HDM-II, so that one has at disposal more possibilities to search for $H^{ \pm}$states at current and future colliders, ideally enabling one to distinguish between different Higgs models of EWSB. We have then concentrated our analysis to the case of the LHC and showed that the production rates of charged Higgs bosons at the LHC is sensitive to the modifications of the Higgs boson couplings. Finally, we have determined the number of events for the most promising LHC signatures of a $H^{ \pm}$belonging to a 2HDM-III, for both $c \bar{b} \rightarrow H^{+}+$c.c. and $q \bar{q} \rightarrow \bar{t} b H^{+}+$c.c. scatterings (the latter affording larger rates than the former). Armed with these results, we are now in a position to carry out a detailed study of signal and background rates, in order to determine the precise detectability level of each signature. However, this is beyond the scope of present work and will be the subject of a future publication.

\section{References}

[1] Kanemura S, Moretti S, Mukai Y, Santos R and Yagyu K 2009 (arXiv:0901.0204 [hep-ph])

[2] Babu K S and Kolda C F 1999 Phys. Lett. B 45177 (arXiv:hep-ph/9811308)

[3] Diaz-Cruz J L, Noriega-Papaqui R and Rosado A 2005 Phys. Rev. D 71015014 (arXiv:hep-ph/0410391)

[4] Diaz-Cruz J L, Hernandez-Sanchez J, Moretti S, Noriega-Papaqui R and Rosado A 2009 Phys. Rev. D 79095025 (arXiv:0902.4490 [hep-ph])

[5] Fritzsch H and Xing Z Z 2003 Phys. Lett.B 55563 (arXiv:hep-ph/0212195)

[6] Cheng T P and Sher M 1987 Phys. Rev.D 353484

[7] J. E. Barradas-Guevara, F. Cazarez-Bush, A. Cordero-Cid, O. Felix-Beltran, J. Hernandez-Sanchez and R. Noriega-Papaqui, J. Phys. G 37, 115008 (2010) [arXiv:1002.2626 [hep-ph]].

[8] Borzumati F and Greub C 1999 Phys. Rev.D 59057501 (arXiv:hep-ph/9809438)

[9] Xiao Z J and Guo L 2004 Phys. Rev.D 69014002 (arXiv:hep-ph/0309103)

[10] Chankowski P H Krawczyk M and Zochowski J 1999 Eur. Phys. J. C 11661 (arXiv:hep-ph/9905436) 